\title{
NUMERICAL STUDY OF THE DAVEY-STEWARTSON SYSTEM
}

\author{
Christophe Besse $^{1}$, Norbert J. Mauser ${ }^{2}$ And Hans Peter Stimming ${ }^{3}$
}

\begin{abstract}
We deal with numerical analysis and simulations of the Davey-Stewartson equations which model, for example, the evolution of water surface waves. This time dependent PDE system is particularly interesting as a generalization of the 1-d integrable NLS to 2 space dimensions. We use a time splitting spectral method where we give a convergence analysis for the semi-discrete version of the scheme. Numerical results are presented for various blow-up phenomena of the equation, including blowup of defocusing, elliptic-elliptic Davey-Stewartson systems and simultaneous blowup at multiple locations in the focusing elliptic-elliptic system. Also the modeling of exact soliton type solutions for the hyperbolic-elliptic (DS2) system is studied.
\end{abstract}

Mathematics Subject Classification. 35Q55, 65M12, 65M70, 76B45.

Received: May 5, 2004. Revised: September 22, 2004.

\section{INTRODUCTION}

The Davey-Stewartson (DS) equations model free surface waves subject to the effects of both gravity and capillarity ("Gravity-Capillary waves"). In dimensionless form, the DS equations read

$$
\begin{aligned}
& i \partial_{t} u+\lambda \partial_{x}^{2} u+\partial_{y}^{2} u=\nu|u|^{2} u+u \partial_{x} \phi, \\
& \quad \alpha \partial_{x}^{2} \phi+\partial_{y}^{2} \phi=\chi \partial_{x}\left(|u|^{2}\right), \quad(x, y) \in \mathbb{R}^{2}, t \in \mathbb{R} .
\end{aligned}
$$

Here $u$ is the (complex) amplitude of the wave and $\phi$ the (real) velocity potential of the wave movement. The parameters $\alpha$ and $\lambda$ can have both signs, according to which the system can be classified as elliptic-elliptic (E-E), elliptic-hyperbolic (E-H), hyperbolic-elliptic (H-E) and hyperbolic-hyperbolic (H-H).

The equations can be viewed as a generalization of the cubic Nonlinear Schrödinger equation (NLS)

$$
i \partial_{t} u+\Delta u=\nu|u|^{2} u
$$

Like the 1-d cubic NLS, the 2-d Davey-Stewartson system is (for specific values of the parameters) integrable by inverse scattering methods $[1,3]$. With respect to finite time blowup, the focusing cubic NLS is the critical

\footnotetext{
Keywords and phrases. Nonlinear Schrödinger type equation, surface wave, time-splitting spectral scheme, finite time blowup.

${ }^{1}$ Laboratoire MIP, UMR 5640, Université Paul Sabatier, 118 Route de Narbonne, 31062 Toulouse Cedex, France.

e-mail: besse@mip.ups-tlse.fr

${ }^{2}$ Wolfgang Pauli Institute c/o Fakultät f. Math., Universität Wien, Nordbergstr. 15, A 1090 Wien, Austria.

e-mail: mauser@courant.nyu.edu

${ }^{3}$ Wolfgang Pauli Institute, Wien and ENS Lyon, France. e-mail: hans.peter.stimming@univie.ac.at
} 
case in 2 space dimensions, and hence an interesting question is given by the blowup behaviour of (focusing) DS equations and its relations to the NLS case.

We use a time-splitting spectral scheme that has been recently developed and applied to a large class of nonlinear Schrödinger type equations $[4,5]$. This scheme has proven to be a quite efficient method and a convenient tool also for the numerical study of "semiclassical limits". We have developed a parallel version of the scheme that also allows for 3-d semiclassical simulations of time dependent NLS $[4,5]$.

In the present work we show numerical results for the blowup of focusing E-E equations and for exact soliton type solutions of the H-E system. We add a study of multi-focusing of the E-E system and an investigation of blowup in the H-E system, which are treated for the first time in this work (to our knowledge).

The paper is organized as follows: in the rest of Section 1, we give an instructive short summary of the physical motivation of equation (1.1) in the context of water waves. In Section 2, we give a convergence proof for the time splitting method and a discussion of the method we apply here. In the third section we present numerical results; in Section 3.1 results on the H-E system and in Section 3.2 on the focusing and defocusing E-E system.

\subsection{Derivation of the model}

We briefly sketch the derivation of the DS equations in the context of water surface waves as it was done by Ablowitz and Segur [2] and Djordjević and Redekopp [10]. The goal is to describe a surface wave in a situation where gravity and the capillarity of the fluid both contribute to the motion on a comparable scale. One considers a non-viscous, incompressible fluid over a flat ground, and studies the free surface flow of this fluid. The fluid is subject to gravity and surface tension forces and is assumed to be initially at rest at height $h$. For small perturbations of the surface, the equations for the motion of the fluid can be linearized around the rest state and read:

$$
\begin{aligned}
\partial_{x}^{2} \phi+\partial_{y}^{2} \phi+\partial_{z}^{2} \phi & =0, & & -h<z<\zeta \\
\partial_{t} \zeta-\partial_{z} \phi & =0, & & z=\zeta \\
\partial_{t} \phi+g \zeta-T\left(\partial_{x}^{2} \zeta+\partial_{y}^{2} \zeta\right) & =0, & & z=\zeta \\
\partial_{z} \phi & =0, & & z=-h .
\end{aligned}
$$

$\phi(x, y, z ; t)$ is the velocity potential of the fluid, $\zeta(x, y ; t)$ the height of the free surface and $T>0$ the relation between the surface tension coefficient and the fluid density.

We look for solutions in form of a plane wave, so the following ansatz is made:

$$
(\phi, \zeta)=\left(f(z) \mathrm{e}^{\mathrm{i}<\kappa, X>-i \omega t}, g \mathrm{e}^{\mathrm{i}<\kappa, X>-i \omega t}\right)
$$

where in the inner product $\langle.,$.$\rangle the vectors \kappa$ and $X$ stand for $\kappa=(k, l), X=(x, y)$. Inserting into the equations (1.3)-(1.6), the dispersion relation for this wave is obtained:

$$
\omega=\left(g \kappa_{0}\left(1+\frac{\kappa_{0}^{2} T}{g}\right) \tanh \kappa_{0} h\right)^{1 / 2}
$$

with $\kappa_{0}=\|\kappa\|$.

The solution of (1.3)-(1.6) is expanded in uniform waves with small amplitudes. Initially the free surface $\zeta$ is perturbed by a uniform wave that has wave number $k_{0}$ and is directed in $x$-direction only, with height $\varepsilon$, which is small compared to $h$ :

$$
\zeta(x, y, t=0)=\varepsilon \frac{i \omega}{g(1+\tilde{T})}\left(A(\varepsilon x, \varepsilon y) \mathrm{e}^{\mathrm{i} k_{0} x}-\overline{A(\varepsilon x, \varepsilon y)} \mathrm{e}^{-i k_{0} x}\right)
$$


where $\tilde{T}=k_{0}^{2} T / g$. The value of the frequency $\omega$ is uniquely determined by the value of $k_{0}$ by the relation (1.7) (note that in this situation $\kappa_{0}=k_{0}$ ). The solution $(\phi, \zeta)$ is then expanded in $\varepsilon$ as well as in uniform waves in $x$-direction $\mathrm{e}^{\mathrm{i}(k x-\omega t)}$ obeying the above mentioned dispersion relation. To get a closed model the following key assumptions are made:

- the initial wave amplitude is small: $\varepsilon \ll 1$, and moreover the dimensionless depth $k h$ is such that the shallow water limit is excluded, so $\varepsilon \ll(k h)^{2}$ is assumed;

- slowly varying modulation $\frac{\delta k}{k_{0}} \ll 1$; and

- the wave is mostly mono-dimensional: $\frac{\left|k_{y}\right|}{k_{0}} \ll 1$; and in addition

- these three effects are in balance to each other such that $\frac{\left|k_{y}\right|}{k_{0}}=\mathrm{O}(\varepsilon)$ and $\frac{\delta k}{k_{0}}=\mathrm{O}(\varepsilon)$.

Then slow variables are introduced

$$
\xi=\varepsilon\left(x-C_{g} t\right), \quad \eta=\varepsilon y, \quad \tau=\varepsilon^{2} t,
$$

where $C_{g}$ is the group velocity defined by

$$
C_{g}=\frac{\partial \omega}{\partial k}=C_{p}\left\{\frac{\sigma+k h\left(1-\sigma^{2}\right)}{2 \sigma}+\frac{\tilde{T}}{1+\tilde{T}}\right\}
$$

(where $\kappa_{0}$ is replaced by $k$ in formula $(1.7)$ ), and $C_{p}$ the phase velocity such that

$$
C_{p}=\frac{\omega}{k} \quad \text { and } \quad \sigma=\tanh k h .
$$

Inserting the expansion and the slow variables into equations (1.3)-(1.6) and sorting by powers of $\varepsilon$ finally leads to the DS equations:

$$
\begin{aligned}
i \partial_{t} \psi+\lambda \partial_{\xi}^{2} \psi+\mu \partial_{\eta}^{2} \psi & =\nu|\psi|^{2} \psi+\nu_{1} \psi \partial_{\xi} \phi \\
\alpha \partial_{\xi}^{2} \phi+\partial_{\eta}^{2} \phi & =\beta \partial_{\xi}\left(|\psi|^{2}\right),
\end{aligned}
$$

where

$$
\begin{aligned}
\lambda & =\frac{1}{2} \omega^{\prime \prime}(k), \quad \mu=\omega^{\prime}(k)=\frac{C_{g}}{2 k}, \\
\nu & =\frac{k^{4}}{4 \omega}\left[\frac{\left(1-\sigma^{2}\right)\left(9-\sigma^{2}\right)+\tilde{T}\left(2-\sigma^{2}\right)\left(7-\sigma^{2}\right)}{\sigma^{2}-\tilde{T}\left(3-\sigma^{2}\right)}+8 \sigma^{2}-2\left(2-\sigma^{2}\right)^{2}(1+\tilde{T})-\frac{3 \sigma^{2} \tilde{T}}{1+\tilde{T}}\right], \\
\nu_{1} & =\frac{k^{2}}{\omega C_{p}}\left[1+\frac{1}{2} \frac{C_{g}}{C_{p}}\left(1-\sigma^{2}\right)(1+\tilde{T})\right], \\
\alpha & =1-C_{g}^{2} / g h, \quad \beta=\frac{k^{2} C_{p}}{g h}\left[\frac{C_{g}}{C_{p}}\left(1-\sigma^{2}\right)+\frac{2 \tilde{T}}{1+\tilde{T}}\right] .
\end{aligned}
$$

By rescaling, this is equivalent to (1.1).

The nature of equations (1.8)-(1.9) changes completely with the sign of $\alpha=1-M^{2}$, where the "Mach number" $M$ is defined as $M=C_{g} / \sqrt{g h}$ and describes the ratio of the wave packet group velocity (which increases with the relative importance of the surface tension) to the phase velocity of the mean field. The motion of the wave packet is said to be "subsonic" when $\alpha>0$ and "supersonic" when $\alpha<0$. For $\alpha>0$, (1.9) is elliptic and the boundary conditions for $\phi$ are $\phi \rightarrow 0$ for $\xi^{2}+\eta^{2} \rightarrow \infty$, and if $\alpha<0$, (1.9) becomes hyperbolic and the boundary conditions for $\phi$ consist in prescribing the flow far upstream in the characteristic 
directions of equation (1.9). The necessary boundary conditions for $\psi$ are the same for both signs, $\psi(\xi, \eta, \tau) \rightarrow 0$ for $\xi^{2}+\eta^{2} \rightarrow \infty$. Depending on the sign of $\lambda$ ( $\mu$ is always positive) the main part of the linear operator in (1.8) is either elliptic of hyperbolic. According to these signs, Ghidaglia and Saut introduced the classification of DS systems as elliptic-elliptic (E-E), elliptic-hyperbolic (E-H), hyperbolic-elliptic (H-E) and hyperbolic-hyperbolic $(\mathrm{H}-\mathrm{H})$, the latter case not occurring in the context of water surface waves.

In the deep water limit $k h \rightarrow \infty$, the system (1.8)-(1.9) reduces to the cubic NLS:

$$
i \partial_{t} \psi+\lambda_{\infty} \partial_{\xi}^{2} \psi+\mu_{\infty} \partial_{\eta}^{2} \psi=\chi_{\infty}|\psi|^{2} \psi
$$

In the long wavelength limit $k h \rightarrow 0$, the parameters in the system (1.8)-(1.9) (resp. (1.1)) become

$$
\mu=\nu_{1}=\beta=1, \alpha=\nu=\sigma_{1}, \lambda=-\sigma_{1} \quad \text { whith } \sigma_{1}=\operatorname{sgn}\left(\frac{1}{3}-\frac{T}{g h^{2}}\right) .
$$

In this case, the system has the remarkable property of being integrable by inverse scattering, and is called "DS1" or "DS2" system according to the value of $\sigma_{1}$.

DS equations are also used in plasma physics for describing the propagation of plasma waves in strong magnetic fields. This derivation is due to Nishinari et al. [19].

\subsection{Invariants and existence results of the DS system}

For the following invariance properties to hold we need to assume that the solution $(u, \phi)$ of $(1.1)$ is sufficiently smooth and decaying at infinity. This restricts the result to the case $\alpha>0$ where the equation for $\phi$ is of elliptic type. If this assumption is satisfied, then the following functionals are independent of time:

$$
\begin{aligned}
N(u) & =\int_{\mathbb{R}^{2}}|u|^{2} \mathrm{~d} x \mathrm{~d} y \\
J_{x}(u) & =\int_{\mathbb{R}^{2}}\left(u \overline{\partial_{x} u}-\bar{u} \partial_{x} u\right) \mathrm{d} x \mathrm{~d} y \\
J_{y}(u) & =\int_{\mathbb{R}^{2}}\left(u \overline{\partial_{y} u}-\bar{u} \partial_{y} u\right) \mathrm{d} x \mathrm{~d} y \\
H(u) & =\int_{\mathbb{R}^{2}} \mu\left|\partial_{x} u\right|^{2}+\lambda\left|\partial_{y} u\right|^{2}+\frac{1}{2}\left(\nu|u|^{4}-\nu_{1} \beta\left(\partial_{x} \phi^{2}+\alpha \partial_{y} \phi^{2}\right)\right) \mathrm{d} x \mathrm{~d} y .
\end{aligned}
$$

The (straightforward) proof is omitted here and can be found for example in [22].

A lot of work has been done on the initial value problem, we try to give a short overview of the most important results relevant to our study. For the elliptic-elliptic and hyperbolic-elliptic case, Ghidaglia and Saut [11] proved local existence and uniqueness, and global existence for small data in $L^{2}\left(\mathbb{R}^{2}\right)$. They also proved that if $u_{0}$ belongs to $H^{2}\left(\mathbb{R}^{2}\right)$, one has $u \in \mathcal{C}\left(\left[0, T^{*}\left[; H^{2}\left(\mathbb{R}^{2}\right)\right) \cap \mathcal{C}^{1}\left(\left[0, T^{*}\left[; L^{2}\left(\mathbb{R}^{2}\right)\right)\right.\right.\right.\right.$.

For the elliptic-elliptic equation we can mention that they proved the existence of solutions which blow up in final time. Denote $\Sigma=\left\{v \mid v \in H^{1}\left(\mathbb{R}^{2}\right),\left(x^{2}+y^{2}\right)^{1 / 2} v \in L^{2}\left(\mathbb{R}^{2}\right)\right\}$. Then the following holds true:

(1) an element $v \in \Sigma$ which satisfies $H(v)<0$ exists if and only if $\nu<\max (-\chi, 0)$;

(2) if $\nu \geq \max (-\chi, 0)$, then the solutions of (1.1) are global;

(3) if $\nu<\max (-\chi, 0)$ and the initial data satisfies $H\left(u_{0}\right)<0$, then the maximal solution exists only for a finite time.

A result similar to this one with regards to existence but with restrictions on the parameters $\nu, \beta, \alpha$ was found by Gúzman-Gomez [12].

The elliptic-hyperbolic and hyperbolic-hyperbolic cases present more difficulties. Hayashi and Hirata [15] obtained local existence for E-H in $H^{s}, s>\frac{5}{2}$, and global existence with an additional smallness condition in some higher order Sobolev spaces. An improvement of the local existence result for E-H in $H^{2}$ was done 
by Hayashi [14] in which he is able to drop the smallness condition. For the H-H case, there is an existence result by Hayashi and Saut [16] in spaces of analytic functions decreasing exponentially at infinity.

\section{Time SPlitting METhod FOR DS Systems}

We use the time-splitting spectral method, which has recently been applied to Nonlinear Schrödinger equations $[4,5]$, and proved to be a powerful tool particularly in studies of the semiclassical limit. It goes back to the 70s [13] and has for the first time been applied to the DS systems by White and Weideman [23] in the E-H and H-E cases. The semi-discrete version of the splitting method was recently proved convergent for the Nonlinear Schrödinger equations [8]. In [21], Papanicolaou et al. studied the self-focusing of E-E DS equations by a numeric resolution of the blowup. Besse and Bruneau [7] have successfully applied a finite difference method to the general DS equations, this method uses a time relaxation approach.

In this article, we focus on the case of a subsonic wavepacket, that is the E-E or H-E versions of the DS system. After a rescaling, it is possible to set $\mu=1$. This system can then be written as a single equation for $u$ (see [11]). We set $\phi_{x}=E\left(|u|^{2}\right)$, where the singular integral operator $E$ is defined in Fourier variables by

$$
\widehat{E(f)}\left(\xi_{1}, \xi_{2}\right)=\frac{\xi_{1}^{2}}{\xi_{1}^{2}+\xi_{2}^{2}} \hat{f}\left(\xi_{1}, \xi_{2}\right)
$$

Therefore, the E-E or H-E DS system reduces to a nonlinear, non-local Schrödinger equation

$$
i \partial_{t} u+L_{\lambda} u=\nu|u|^{2} u+\nu_{1} E\left(|u|^{2}\right) u=F(u)
$$

where $L_{\lambda}=\lambda \partial_{\xi}^{2}+\partial_{\eta}^{2}$ with $\lambda= \pm 1$.

\subsection{Convergence of the split-step method}

The idea behind the method is to decouple the nonlinear system into a linear PDE with constant coefficients, which can be discretized in space by a spectral method or by classical finite difference method, and a nonlinear equation which can be solved exactly. Therefore, the split-step method is based on a decomposition of the flow of (2.2).

Indeed, let us define the flow $X^{t}$ of the linear Schrödinger equation

$$
\left\{\begin{array}{l}
i \partial_{t} v+L_{\lambda} v=0, \mathbf{x} \in \mathbb{R}^{2}, t>0 \\
v(\mathbf{x}, 0)=v_{0}(\mathbf{x}), \mathbf{x} \in \mathbb{R}^{2}
\end{array}\right.
$$

and $Y^{t}$ as the flow of the nonlinear differential equation

$$
\left\{\begin{array}{l}
i \partial_{t} w=F(w), \quad \mathbf{x} \in \mathbb{R}^{2}, t>0 \\
w(\mathbf{x}, 0)=w_{0}(\mathbf{x}), \mathbf{x} \in \mathbb{R}^{2}
\end{array}\right.
$$

Then the splitting method consists of combining the two flows $X^{t}$ and $Y^{t}$. The best known methods are the following: the most simple "Lie formula" given by $Z_{L_{1}}^{t}=X^{t} Y^{t}$ (or $Z_{L_{2}}^{t}=Y^{t} X^{t}$ ) and the Strang formula $Z_{S}^{t}=Y^{t / 2} X^{t} Y^{t / 2}\left(\right.$ or $\left.X^{t / 2} Y^{t} X^{t / 2}\right)$.

Using the previously defined flows, we can define a new flow $S^{t}$ which gives the solution of $(2.2)$ as

$$
u(\mathbf{x}, t)=S^{t} u_{0}(\mathbf{x})=X^{t} u_{0}(\mathbf{x})-i \int_{0}^{t} X^{t-s} F(u(\mathbf{x}, s)) \mathrm{d} s .
$$

We use the first Strang formulation $Z_{S}^{t}=Y^{t / 2} X^{t} Y^{t / 2}$ and prove that this scheme is convergent for the DS problem. For a general nonlinear Schrödinger equation, this convergence proof has been done by Besse et al. in [8]. 
Note that their convergence result holds only for initial data belonging to $H^{4}$. For E-E or H-E versions of DS that method does not apply and the proof must be adapted for initial data belonging to $H^{2}$, which is a nontrivial task.

We use extensively the fact that on a time interval $[0, T]$, before a possible blow-up of the solution, $u$ remains bounded in $H^{2}$, and set $R=\sup _{t \in[0, T]}\|u\|_{H^{2}}$.

The main result of this part is

Theorem 2.1. For all $u_{0}$ in $H^{2}$ and for all $0<T<T^{*}$, there exist $C$ and $h_{0}$ such that for all $h \in\left(0, h_{0}\right]$ and for all $n$ with $n h \leq T$ :

$$
\left\|\left(Z_{S}^{h}\right)^{n} u_{0}-S^{n h} u_{0}\right\| \leq C\left(\left\|u_{0}\right\|_{H^{2}}\right) h\left\|u_{0}\right\|_{H^{2}}
$$

To prove this result for a small $h>0$ and all integer $n$ such that $n h \leq T$, we have to estimate the quantity $\left\|\left(Z_{\mathrm{S}}^{h}\right)^{n} u_{0}-S^{n h} u_{0}\right\|$, where $\|\cdot\|$ denotes the $L^{2}$ norm. As noticed in [9], the triangle inequality yields

$$
\left\|\left(Z_{\mathrm{S}}^{h}\right)^{n} u_{0}-S^{n h} u_{0}\right\| \leq \sum_{j=0}^{n-1}\left\|\left(Z_{\mathrm{S}}^{h}\right)^{n-j-1} Z_{\mathrm{S}}^{h} S^{j h} u_{0}-\left(Z_{\mathrm{S}}^{h}\right)^{n-j-1} S^{(j+1) h} u_{0}\right\| .
$$

We prove in Section 2.1.4 that there exists a constant $C_{0}$ such that for $w_{0}$ and $w_{0}^{\prime} \in L^{2}$ and all $t \in[0,1]$,

$$
\left\|Z_{\mathrm{S}}^{t} w_{0}-Z_{\mathrm{S}}^{t} w_{0}^{\prime}\right\| \leq\left(1+C_{0} t\right)\left\|w_{0}-w_{0}^{\prime}\right\|
$$

Therefore

$$
\left\|\left(Z_{\mathrm{S}}^{h}\right)^{n} u_{0}-S_{\mathrm{S}}^{n h} u_{0}\right\| \leq \sum_{j=0}^{n-1}\left(1+C_{0} h\right)^{n-j-1}\left\|\left(Z_{\mathrm{S}}^{h}-S_{\mathrm{S}}^{h}\right) S^{j h} u_{0}\right\| .
$$

Thus we may restrict our study to the case for which at each time step the initial data is the same for the continuous model and the splitting scheme and is equal to $v_{0}=S^{j h} u_{0}$.

\subsubsection{Estimates on the linear flow}

From the definition of the Schrödinger flow, we first have

$$
\dot{X}^{t}=i L_{\lambda} X^{t}=i X^{t} L_{\lambda}
$$

As the flow $X^{t}$ is a unitary group on $H^{2}$, we have

$$
\left\|X^{t} u_{0}\right\|_{H^{2}}=\left\|u_{0}\right\|_{H^{2}} .
$$

\subsubsection{Estimates on the nonlinear flow}

The nonlinear flow is defined as

$$
Y^{t} w=w-i \int_{0}^{t} F\left(Y^{s} w\right) \mathrm{d} s
$$

Note that by a simple calculation, it follows from (2.4) and the explicit form of $F$ that

$$
\left|Y^{t} w\right|=|w|
$$

and with this result, the nonlinear flow can be expressed as

$$
Y^{t} w=\mathrm{e}^{-i\left(\nu|w|^{2}+\nu_{1} E\left(|w|^{2}\right)\right) t} w
$$

Lemma 2.2. For all $t \in[0, T]$,

holds.

$$
\left\|Y^{t} w\right\|_{H^{2}} \leq\|w\|_{H^{2}}
$$


Proof. The application of $F$ is Lipschitz according to Ghidaglia and Saut [11] (p. 485, Lem. 2.1). We denote by $K$ its Lipschitz constant. Equation (2.10) first yields the following $L^{\infty}$ estimate:

$$
\left\|Y^{t} w\right\|_{\infty} \leq\|w\|_{\infty}+K \int_{0}^{t}\left\|Y^{s} w\right\|_{\infty} \mathrm{d} s .
$$

Then the classical Gronwall lemma gives

$$
\left\|Y^{t} w\right\|_{\infty} \leq \mathrm{e}^{K t}\|w\|_{\infty}
$$

On the other hand, (2.10) also leads to an $L^{2}$ estimate:

$$
\left\|Y^{t} w\right\| \leq\|w\|+K \int_{0}^{t}\left\|Y^{s} w\right\| \mathrm{d} s .
$$

For all first order differential operators $D$, it holds that

$$
D Y^{t} w=D w-i \int_{0}^{t} F^{\prime}\left(Y^{s} w\right) D\left(Y^{s} w\right) \mathrm{d} s
$$

and, denoting by $M^{\prime}$ the maximum for $F^{\prime}$, we obtain

$$
\left\|D Y^{t} w\right\| \leq\|D w\|+M^{\prime} \int_{0}^{t}\left\|D Y^{s} w\right\| \mathrm{d} s .
$$

Differentiating once more (here, $\Delta$ denotes the Laplacian operator) leads to

$$
\Delta Y^{t} w=\Delta w-i \int_{0}^{t}\left(F^{\prime \prime}\left(Y^{s} w\right) D\left(Y^{s} w\right)^{2}+F^{\prime}\left(Y^{s} w\right) \Delta Y^{s} w\right) \mathrm{d} s
$$

So

$$
\begin{aligned}
\left\|\Delta Y^{t} w\right\| & \leq\|\Delta w\|+\int_{0}^{t}\left(M^{\prime \prime}\left\|D Y^{s} w\right\|^{2}+M^{\prime}\left\|\Delta Y^{s} w\right\|\right) \mathrm{d} s \\
& \leq\|\Delta w\|+\int_{0}^{t}\left(M^{\prime \prime}\left\|Y^{s} w\right\|_{\infty}+M^{\prime}\right)\left\|Y^{s} w\right\|_{H^{2}} \mathrm{~d} s
\end{aligned}
$$

where the Gagliardo-Nirenberg inequality was applied and $M^{\prime \prime}$ denotes the maximum for $F^{\prime \prime}$. Therefore, by using the $L^{\infty}$ estimate above, there exists a constant $\tilde{c}$ such that

$$
\left\|Y^{t} w\right\|_{H^{2}} \leq\|w\|_{H^{2}}+\tilde{c} \int_{0}^{t}\left(1+\mathrm{e}^{K s}\right)\left\|Y^{s} w\right\|_{H^{2}} \mathrm{~d} s
$$

and by the Gronwall lemma,

$$
\left\|Y^{t} w\right\|_{H^{2}} \leq\|w\|_{H^{2}} \exp \left(\tilde{c} \int_{0}^{t}\left(1+\mathrm{e}^{K s}\right) \mathrm{d} s\right) .
$$

For $t \leq 1$, there exists a constant $C$ such that $\exp \left(\tilde{c} \int_{0}^{t}\left(1+\mathrm{e}^{K s}\right) \mathrm{d} s\right) \leq C$, and the assertion follows. 
Lemma 2.3. Let $X=L^{\infty}\left([0, T] ; L^{2}\left(\mathbb{R}^{2}\right)\right)$.

(1) Let $w \in X$; then

$$
\|E(w)\|_{X} \leq\|w\|_{X} .
$$

(2) Let $w$ and $w^{\prime} \in B_{X}(0, R)$, where $B_{X}(0, R)$ is the ball centered at 0 of $X$; then

$$
\left\|E\left(|w|^{2}\right) w-E\left(\left|w^{\prime}\right|^{2}\right) w^{\prime}\right\|_{X} \leq \operatorname{Lip}_{E}(R)\left\|w-w^{\prime}\right\|_{X},
$$

where $\operatorname{Lip}_{E}(R)$ is the Lipschitz constant of the map $E$ on the ball $B_{X}(0, R)$.

(3) Let $w \in X$; then

$$
\left|E\left(|w|^{2}\right) w\right| \leq|w|^{2}|w|
$$

Proof.

(1) Let $\mathcal{F}^{-1}$ denote the inverse Fourier transform. Then

$$
\begin{aligned}
\|E(w)\|_{X} & =\left\|\mathcal{F}^{-1}\left(\frac{\xi_{1}^{2}}{\xi_{1}^{2}+\xi_{2}^{2}} \hat{w}\left(\xi_{1}, \xi_{2}\right)\right)\right\|_{X} \\
& =\left\|\int \mathrm{e}^{\mathrm{i}<x, \xi>} \frac{\xi_{1}^{2}}{\xi_{1}^{2}+\xi_{2}^{2}} \hat{w}\left(\xi_{1}, \xi_{2}\right) \mid \mathrm{d} \xi\right\|_{X} \\
& \leq\left\|\int \mathrm{e}^{\mathrm{i}<x, \xi>} \hat{w}\left(\xi_{1}, \xi_{2}\right) \mid \mathrm{d} \xi\right\|_{X} \\
& \leq\|w\|_{X} .
\end{aligned}
$$

(2)

$$
\begin{aligned}
\| E\left(|w|^{2}\right) w & -E\left(\left|w^{\prime}\right|^{2}\right) w^{\prime} \|_{X} \\
& =\left\|E\left(|w|^{2}\right)\left(w-w^{\prime}\right)+w^{\prime}\left(E\left(|w|^{2}\right)-E\left(\left|w^{\prime}\right|^{2}\right)\right)\right\|_{X} \\
& \leq\left\|E\left(|w|^{2}\right)\right\|_{X}\left\|w-w^{\prime}\right\|_{X}+\left\|w^{\prime}\right\|_{X}\left\|E\left(|w|^{2}\right)-E\left(\left|w^{\prime}\right|^{2}\right)\right\|_{X} \\
& \leq\left\|E\left(|w|^{2}\right)\right\|_{X}\left\|w-w^{\prime}\right\|_{X}+\left\|w^{\prime}\right\|\left\|E\left(|w|^{2}-\left|w^{\prime}\right|^{2}\right)\right\| .
\end{aligned}
$$

As $H^{2}\left(\mathbb{R}^{2}\right)$ is an algebra and thanks to $(2.14)$, we have

$$
\left\|E\left(|w|^{2}\right) w-E\left(\left|w^{\prime}\right|^{2}\right) w^{\prime}\right\|_{X} \leq\|w\|_{X}^{2}\left\|w-w^{\prime}\right\|_{X}+\left\|w^{\prime}\right\|_{X}\left\||w|^{2}-\left|w^{\prime}\right|^{2}\right\|_{X} .
$$

As $|w|^{2}-\left|w^{\prime}\right|^{2}=\left(|w|+\left|w^{\prime}\right|\right)\left(|w|-\left|w^{\prime}\right|\right)$, we finally have

$$
\left\|E\left(|w|^{2}\right) w-E\left(\left|w^{\prime}\right|^{2}\right) w^{\prime}\right\|_{X} \leq 3 R^{2}\left\|w-w^{\prime}\right\|_{X} .
$$

(3) The proof is similar to the previous one.

\subsubsection{Local errors}

Lemma 2.4. Let $v_{0} \in H^{2}, 0 \leq t \leq 1$ and $k \in\{1,2\}$.

(1) If $v_{0} \in B_{H^{2}}(0, R)$, then $\left\|Z_{L_{k}} v_{0}\right\| \leq C(R)$.

(2) There exist two constants $C_{1}$ and $C_{2}$ such that

$$
\left\|\left(Z_{L_{k}}^{t}-S^{t}\right) v_{0}\right\| \leq C_{1} \int_{0}^{t}\left\|\left(Z_{L_{k}}^{\sigma}-S^{\sigma}\right) v_{0}\right\| \mathrm{d} \sigma+C_{2}\left\|v_{0}\right\|_{H^{2}} \frac{t^{2}}{2} .
$$


Proof. The proof is similar for the two flows $Z_{L_{1}}$ and $Z_{L_{2}}$, so we only treat the first one.

Let $v=Z_{L_{1}}^{t} v_{0}$ the splitting solution and $u=S^{t} v_{0}$ the exact solution. Thanks to the definitions of the flows, we get

$$
\begin{aligned}
& v=X^{t} v_{0}-i \int_{0}^{t} F\left(Y^{\sigma} X^{t} v_{0}\right) \mathrm{d} \sigma \\
& u=X^{t} v_{0}-i \int_{0}^{t} X(t-\sigma) F(u(\sigma)) \mathrm{d} \sigma .
\end{aligned}
$$

We first estimate if $v$ remains bounded if $v_{0}$ is taken in the ball $B_{H^{2}}(0, R)$. Using the fact that $H^{2}\left(\mathbb{R}^{2}\right)$ is an algebra and relations (2.9), (2.12) and (2.16), we have

$$
\begin{aligned}
\|v\|_{H^{2}} & \leq\left\|v_{0}\right\|_{H^{2}}+\int_{0}^{t}\left|\nu+\nu_{1}\right|\left\|Y^{\sigma} X^{t} v_{0}\right\|_{H^{2}}^{3} \mathrm{~d} \sigma \\
& \leq R+t R^{3}
\end{aligned}
$$

We now express the difference between the splitting solution and the exact solution.

$$
\|v-u\|=\left\|i \int_{0}^{t} X(t-\sigma)[F(u(\sigma))-F(v(\sigma))]+i \int_{0}^{t} X(t-\sigma) F\left(Y^{\sigma} X^{\sigma} v_{0}\right)-F\left(Y^{\sigma} X^{t} v_{0}\right) \mathrm{d} \sigma\right\| .
$$

Using the fact that $v$ and $u$ remain bounded if $v_{0} \in B_{H^{2}}(0, R)$, we use the Lipschitz constant of $F$ and get

$$
\|v-u\| \leq \operatorname{Lip}_{F}(R) \int_{0}^{t}\|(v-u)(\sigma)\|_{H^{2}} \mathrm{~d} \sigma+\int_{0}^{t}\left\|X(t-\sigma) F\left(Y^{\sigma} X^{\sigma} v_{0}\right)-F\left(Y^{\sigma} X^{t} v_{0}\right)\right\| \mathrm{d} \sigma .
$$

Let us define

$$
R_{1}(\sigma)=\underbrace{X(t-\sigma) F\left(Y^{\sigma} X^{\sigma} v_{0}\right)-F\left(Y^{\sigma} X^{\sigma} v_{0}\right)}_{A}+\underbrace{F\left(Y^{\sigma} X^{\sigma} v_{0}\right)-F\left(Y^{\sigma} X^{t} v_{0}\right)}_{B} .
$$

We have, thanks to $(2.8)$,

$$
A=\int_{\sigma}^{t} \dot{X}(\tau-\sigma) F\left(Y^{\sigma} X^{\sigma} v_{0}\right) \mathrm{d} \tau=\int_{\sigma}^{t} i X L_{\lambda} F\left(Y^{\sigma} X^{\sigma} v_{0}\right) \mathrm{d} \tau
$$

Therefore $\|A\| \leq(t-\sigma)\left\|L_{\lambda} F\left(Y^{\sigma} X^{\sigma} v_{0}\right)\right\| \leq(t-\sigma)\left\|F\left(Y^{\sigma} X^{\sigma} v_{0}\right)\right\|_{H^{2}}$. Using again the Lipschitz constant of $F$ and properties of the flows $X^{t}$ and $Y^{t}$ we get

$$
\|A\| \leq \operatorname{Lip}_{F}(R)\left\|u_{0}\right\|_{H^{2}}
$$

The estimates on the term $\mathrm{B}$ follow the same routine

$$
\|B\| \leq \operatorname{Lip}_{F}(R)\left\|X^{t}\left(X^{\sigma-t}-I\right) v_{0}\right\| \leq \operatorname{Lip}_{F}(R)\left\|\left(X^{\sigma-t}-I\right) v_{0}\right\|
$$

Thus we have again $\|B\| \leq \operatorname{Lip}_{F}(R)\left\|u_{0}\right\|_{H^{2}}$.

The term $R_{1}$ is therefore bounded $\left\|R_{1}(\sigma)\right\| \leq C(\sigma-t)\left\|v_{0}\right\|_{H^{2}}$ and the difference between the splitting solution and the exact solution becomes

$$
\left\|\left(Z_{L_{1}}^{t}-S^{t}\right) v_{0}\right\| \leq C_{1} \int_{0}^{t}\left\|\left(Z_{L_{1}}^{\sigma}-S^{\sigma}\right) v_{0}\right\| \mathrm{d} \sigma+C_{2}\left\|v_{0}\right\|_{H^{2}} \frac{t^{2}}{2} .
$$

We proceed in a same way for the term $\left\|\left(Z_{L_{2}}^{t}-S^{t}\right) v_{0}\right\|$. 
From an adaption of the Gronwall lemma (cf. [8]) we get

Lemma 2.5. For $k \in\{1,2\}$ and $0 \leq t \leq 1$, the relation (2.17) yields

$$
\left\|\left(Z_{L_{k}}^{t}-S^{t}\right) v_{0}\right\| \leq C \frac{t^{2}}{2}
$$

\subsubsection{Lipschitz properties}

Lemma 2.6. Let $w_{0}$ and $w_{0}^{\prime} \in B_{H^{2}}(0, R), 0 \leq t \leq 1$. Then there exists a constant $C$ depending only on $F$ such that

$$
\left\|Z_{S}^{t} w_{0}-Z_{S}^{t} w_{0}^{\prime}\right\| \leq(1+C t)\left\|w_{0}-w_{0}^{\prime}\right\| .
$$

Proof. As the flow $Z_{S}^{t}$ is defined through the relation $Z_{S}^{t}=Y^{t / 2} X^{t} Y^{t / 2}$, we have

$$
w=Z_{S}^{t} w_{0}=X^{t} Y^{t / 2} w_{0}-i \int_{0}^{t / 2} F\left(Y^{s} X^{t} Y^{t / 2} w_{0}\right) \mathrm{d} s .
$$

Therefore

$$
Z_{S}^{t} w_{0}-Z_{S}^{t} w_{0}^{\prime}=X^{t} Y^{t / 2} w_{0}-X^{t} Y^{t / 2} w_{0}^{\prime}-i \int_{0}^{t / 2} F\left(Y^{s} X^{t} Y^{t / 2} w_{0}\right)-F\left(Y^{s} X^{t} Y^{t / 2} w_{0}^{\prime}\right) \mathrm{d} s .
$$

Using again properties $(2.9),(2.12)$ and the Lipschitz constant of $F$, we have

$$
\left\|Z_{S}^{t} w_{0}-Z_{S}^{t} w_{0}^{\prime}\right\| \leq\left\|w_{0}-w_{0}^{\prime}\right\|+\operatorname{Lip}_{F}(R) t\left\|w_{0}-w_{0}^{\prime}\right\| .
$$

\subsubsection{Order estimate}

We are now able to prove the Theorem 2.1. Thanks to Lemma 2.5, we can compute

$$
\begin{aligned}
\left\|\left(Z_{S}^{h}-S^{h}\right) v_{0}\right\| & =\left\|Z_{L_{1}}^{h / 2} Z_{L_{2}}^{h / 2} v_{0}-S^{h / 2} S^{h / 2} v_{0}\right\| \\
& =\left\|Z_{L_{1}}^{h / 2} Z_{L_{2}}^{h / 2} v_{0}-S^{h / 2} Z_{L_{2}}^{h / 2} v_{0}+S^{h / 2} Z_{L_{2}}^{h / 2} v_{0}-S^{h / 2} S^{h / 2} v_{0}\right\| \\
& \leq\left\|\left(Z_{L_{1}}^{h / 2}-S^{h / 2}\right) Z_{L_{2}}^{h / 2} v_{0}\right\|+\left\|S^{h / 2}\left(Z_{L_{2}}^{h / 2}-S^{h / 2}\right) v_{0}\right\| \\
& \leq C^{\prime} \frac{h^{2}}{4}\left\|v_{0}\right\|_{H^{2}},
\end{aligned}
$$

where the constant $C^{\prime}$ depends only on the Lipschitz constant of $F$. Using now the inequality (2.7), we get

$$
\begin{aligned}
\left\|\left(Z_{S}^{h}\right)^{n} u_{0}-S^{h n} u_{0}\right\| & \leq \frac{C^{\prime}}{4}\left\|u_{0}\right\|_{H^{2}} \sum_{j=0}^{n-1} \exp (C h)^{n-j-1} h^{2} \\
& \leq \frac{C^{\prime}}{4}\left\|u_{0}\right\|_{H^{2}} \exp (C T) n h^{2} \\
& \leq C^{\prime \prime}\left\|u_{0}\right\|_{H^{2}} h .
\end{aligned}
$$

Therefore the split-step scheme is convergent.

\subsection{Time-splitting spectral scheme}

The crucial advantages of the time-splitting spectral method are that it is fully explicit, unconditionally stable, time reversible, and time-transverse invariant [4]. 
We use the Strang splitting method $Z_{S}^{t}=Y^{t / 2} X^{t} Y^{t / 2}$. The linear flow $X^{t}$ is solved by a spectral Fourier method and the nonlinear flow $Y^{t}$ by exact integration via formula (2.1).

We choose a square domain $[a, b]^{2}$ and the same grid size in $\mathrm{x}$ - and y-direction, $\Delta x=\Delta y=(b-a) / M$ for an even, positive integer $M$. The time step is $\Delta t$ and the grid points are $\left(x_{j}, y_{k}\right)=(a+j \Delta x, a+k \Delta x)$, $t_{n}=n \Delta t$. The approximation of $u\left(x_{j}, y_{k}, t_{n}\right)$ is denoted $u_{j k}^{n}$, and $u^{n}$ is the solution matrix at time $t_{n}$ with components $u_{j k}^{n}$. We impose periodic boundary conditions for a sufficiently fast decaying $\psi$ in order to apply the spectral method.

In detail, the method is:

$$
\begin{aligned}
\mathrm{u}_{j k}^{*} & =\frac{1}{M^{2}} \sum_{l=-M / 2}^{M / 2-1} \sum_{m=-M / 2}^{M / 2-1} \mathrm{e}^{-i\left(\alpha \mu_{l}^{2}+\mu_{m}^{2}\right) \Delta t / 2} \widehat{\left(u^{n}\right)_{l m}} \mathrm{e}^{\mathrm{i}\left(\mu_{l}\left(x_{j}-a\right)+\mu_{m}\left(x_{k}-a\right)\right)}, \\
E_{j k}^{*} & =\frac{1}{M^{2}} \sum_{-M / 2 \leq l, m \leq M / 2-1,(l, m) \neq(0,0)} \frac{\beta \nu_{1} \mu_{l}^{2}}{\mu_{l}^{2}+\mu_{m}^{2}} \widehat{\left(\left|u^{*}\right|^{2}\right)_{l m}} \mathrm{e}^{\mathrm{i}\left(\mu_{l}\left(x_{j}-a\right)+\mu_{m}\left(x_{k}-a\right)\right)}, \\
u_{j k}^{* *} & =\mathrm{e}^{-i\left(E_{j k}^{*}+\nu\left|u_{j k}^{*}\right|^{2}\right) \Delta t} u_{j k}^{*}, \\
\mathrm{u}_{j k}^{n+1} & =\frac{1}{M^{2}} \sum_{l=-M / 2}^{M / 2-1} \sum_{m=-M / 2}^{M / 2-1} \mathrm{e}^{-i\left(\alpha \mu_{l}^{2}+\mu_{m}^{2}\right) \Delta t / 2} \widehat{\left(u^{* *}\right)_{l m}} \mathrm{e}^{\mathrm{i}\left(\mu_{l}\left(x_{j}-a\right)+\mu_{m}\left(x_{k}-a\right)\right)}, \\
& j, k=0,1,2, \cdots, M-1 .
\end{aligned}
$$

Here $\widehat{U}_{l, m}(l, m=-M / 2, \cdots, M / 2-1)$ is the 2-D discrete Fourier transform of a (periodic) matrix $U$ :

$$
\begin{array}{r}
\widehat{U}_{l, m}=\sum_{j=0}^{M-1} \sum_{k=0}^{M-1} U_{j k} \mathrm{e}^{-i\left(\mu_{l}\left(x_{j}-a\right)+\mu_{m}\left(x_{k}-a\right)\right)} \\
\mu_{l}=\frac{2 \pi}{b-a} l, \quad l, m=-\frac{M}{2}, \cdots, \frac{M}{2}-1
\end{array}
$$

In order to be able to run simulations with a sufficiently fine discretization, we adapted the code for the use on a distributed memory parallel machine. We used the cluster "Schrödinger 2" of the University of Vienna, which features 192 Pentium IV processors with 1 GB memory per node, linked by a switched gigabit network. (For more details on the parallel code, see [5]). We used the parallel version of the scheme in particular for the study of blow-up in E-E systems.

\section{NumericAl RESUlts}

\subsection{Hyperbolic-elliptic DS system}

\subsubsection{Exact solutions}

The hyperbolic-elliptic DS system with the parameter set (1.10) and $\sigma_{1}=1$, called "DS2", is an integrable system. Arkadiev et al. [3] proved the existence of a class of exact solutions by inverse scattering methods. These show localized structures getting displaced without dispersion and for this reason are said to be of "soliton type". To formulate these solutions we first rewrite the equation as

$$
\begin{gathered}
i u_{t}+\partial_{x}^{2} u-\partial_{y}^{2} u=-2 \chi|u|^{2} u-u \phi_{x}, \\
\Delta \phi=-4 \chi\left(|u|^{2}\right)_{x} .
\end{gathered}
$$




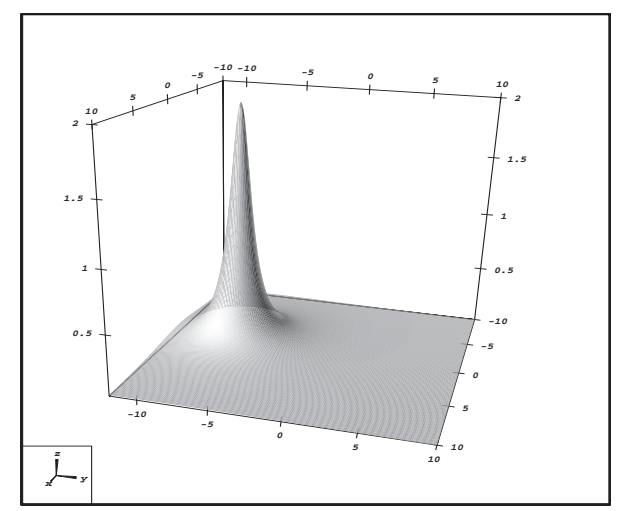

Figure 1. Exact solution at time $t_{0}=-3.5$.

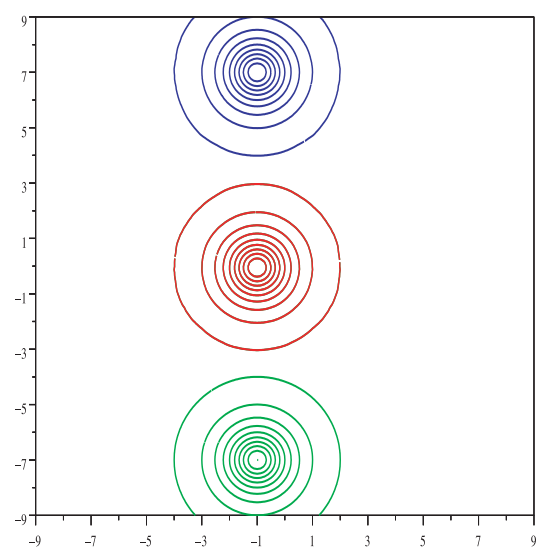

FiguRE 2. Traveling "soliton" at times $t=-3.5,0,3.5$.

Then the exact solution is

$$
u(x, y, t)=\frac{2 \bar{\nu} \exp \left(x(\lambda-\bar{\lambda})+i y(\lambda+\bar{\lambda})+2 i t\left(\lambda^{2}+\bar{\lambda}^{2}\right)\right)}{|x+i y+\mu-2 i \lambda t|^{2}-\chi|\nu|^{2}}
$$

where $\nu, \mu, \lambda \in \mathbb{C}$ are parameters. We choose $\chi=-1$ and $\nu=\mu=\lambda=1$. With this choice, the expression of the solution is

$$
u(x, y, t)=\frac{4 \exp (i y+2 i t)}{(x+1)^{2}+(y-2 t)^{2}+1} .
$$

Figure 1 shows the initial data at $t_{0}=-3.5$, it is a hump centered at $x=-1, y=-7=2 \cdot t_{0}$. This solution has only geometric decay towards infinity, which poses a problem for our scheme which needs to rely on a rapid decay of solutions to prevent errors from the artificial periodic boundary conditions. To prevent such errors we need to take a rather large domain, we choose $\Omega=[-16,16]^{2}$. Figure 2 shows contour plots of $|u|$ at different times during the evolution. We can see that the "soliton structure" is traveling in $y$-direction at speed 2 as required. Also the shape of the solution is preserved accurately. In Figure 3 we show a numeric test of the conserved quantities mentioned above. The wave energy $N(u)$ is conserved exactly up to machine precision, so we do not show a picture for that result. The $L^{\infty}$-norm of $u$, which should be constant due to the exact solution formula, shows slight oscillations while the current density ( $x$-component) $J_{x}$ is almost exactly conserved. For the conservation of the Hamiltonian $H(x)$ we discovered a particular sensitivity to the artificial domain cutoff 
a)

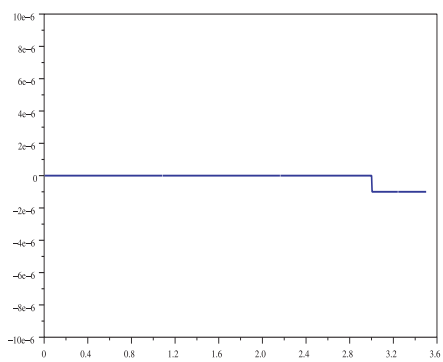

b)

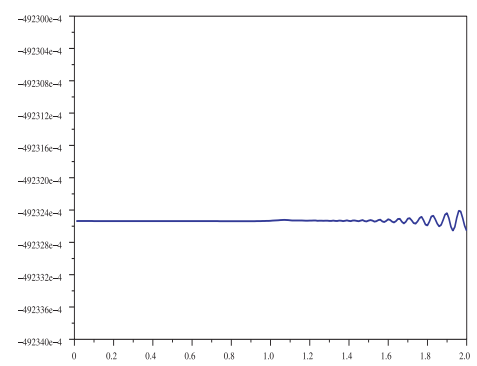

c)

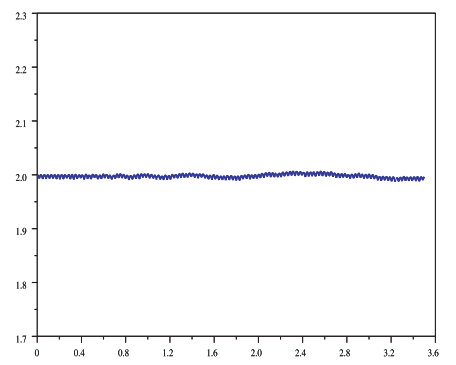

Figure 3. (a): Current density $J(x)$, component in $x$-direction. (b): Hamiltonian of Hyperb.Ell. system. (c): $L^{\infty}-$ norm of $|\psi|^{2} ; \Omega=[-40,40]^{2}$.

when the evolution lasts longer. With a larger domain, in this case $[-40,40]^{2}$, the Hamiltonian is conserved up to 5 digits and time $t \leq 2$.

\subsubsection{Blowup of $H-E$ system}

The next test case for the DS2 equation is the case of a finite time blowup studied by Ozawa [20], where an exact solution is constructed that blows up at a given time. The initial data are $u_{0}(x, y)=\frac{\exp \left(i\left(x^{2}-y^{2}\right)\right)}{1+x^{2}+y^{2}}$, which is a localized lump with algebraic decay (as in the previous case). By the construction we know that the blowup time is $t_{*}=0.25$ and $\|u(t)\|_{L_{\infty}}=1 /(1-4 t)$ holds for he exact solution.

In this test we found the code to be very sensitive to cutoff errors from the artificial boundary conditions. We need to calculate on the domain $[-40,40]^{2}$ to get reasonable results. Figure 4 shows the blowup for three different choices of the resolutions (upper line $\Delta x=0.01953, \Delta t=10^{-4}$, middle line $\Delta x=0.039, \Delta t=2.5 \times 10^{-4}$, lower line $\left.\Delta x=0.0781, \Delta t=5 \times 10^{-4}\right)$, and the exact rate stated above as a dashed line. We can see that, if the discretization is chosen fine enough, we can recover the correct blowup rate. Figure 5 shows the position density close to the blowup time.

\subsection{Elliptic-elliptic DS system}

\subsubsection{Finite time blowup}

In this section we treat the elliptic-elliptic DS system

$$
\begin{aligned}
i u_{t}+\Delta u & =\chi|u|^{2} u+u \phi_{x} \\
\Delta \phi & =-\gamma\left(|u|^{2}\right)_{x} .
\end{aligned}
$$

Finite time blowup of this system was studied by Ghidaglia and Saut [11]. As mentioned in Section 1, (3.1) always leads to blow-up either if $\chi=-1$, for any $\gamma$, or if $\chi=1$ and $\gamma>\chi$. We choose the initial data profile

$$
u_{I}(x, y)=4 \exp \left(\frac{-x^{2}-y^{2}}{4}\right) \text {. }
$$




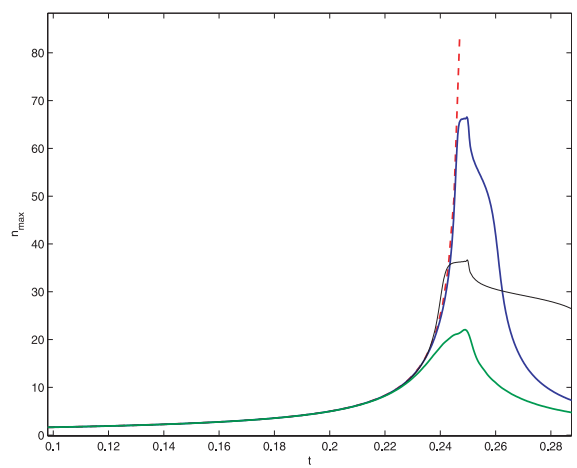

FiguRE $4 . L^{\infty}$-norm of blowup solution for increasingly refined discretizations. Dashed line is analytic blowup rate.

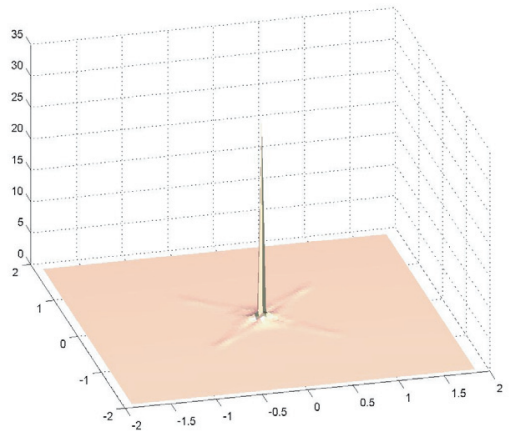

Figure 5. Position density close to blowup.

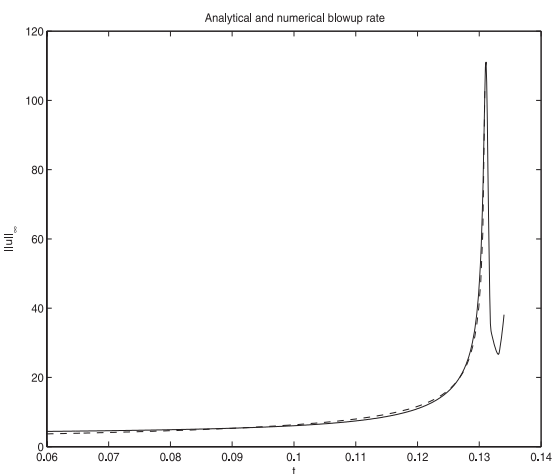

FiguRE $6 . \quad L^{\infty}$ norm of $|\Psi|$ and analytical blowup rate.

First we investigate the case $\chi=-1$ (focusing nonlinearity) and set $\chi=-1, \gamma=1$. This case is analogous to the focusing NLS, since for $\gamma=0$, (3.1) reduces to the focusing cubic NLS, which in two space dimensions is the critical case for finite-time blowup. We expect the blowup mechanism to be similar to the one of that equation. We find that the solution blows up at time $t=0.1311$. Figure 6 shows the $L^{\infty}$-norm of $u$. Papanicolaou et al. [21] 
a)

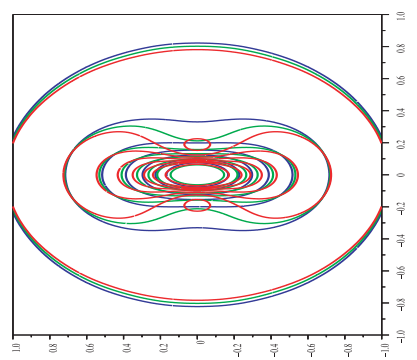

b)

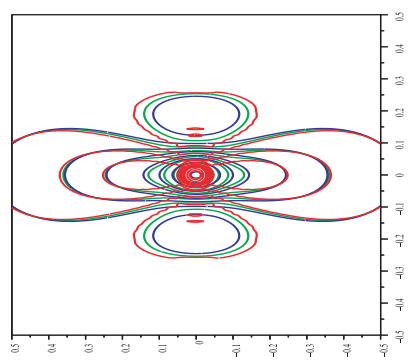

Figure 7. Contour plot of position densities: (a) before blowup, at $t=0.12, t=0.124$, $t=0.128$, (b) close to blowup, at $t=0.1298, t=0.1304, t=0.1308$.

a)

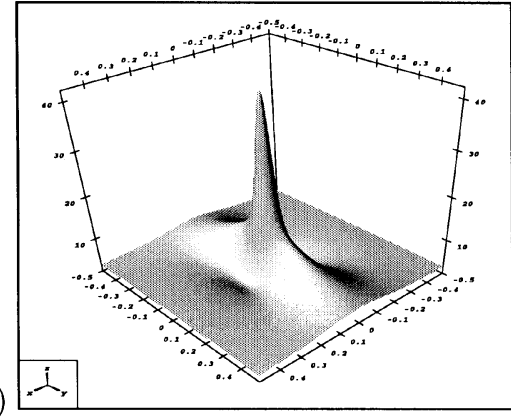

b)
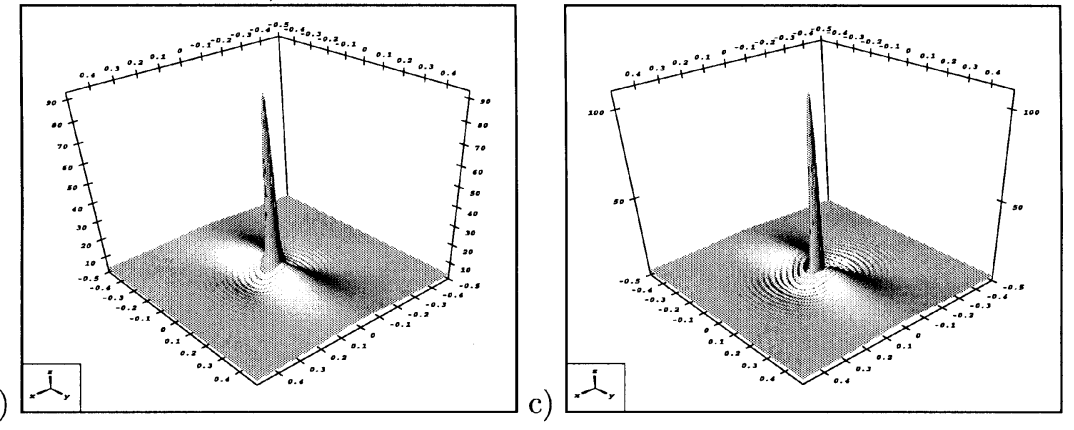

Figure 8. Position density at different times: (a) $t=0.1298$, (b) $t=0.1308$, (c) $t=0.131$.

analytically found that the solution blows up at the rate

$$
L(t) \approx\left(t_{*}-t\right)^{\frac{1}{2}}\left(\ln \ln \frac{1}{t_{*}-t}\right)^{-\frac{1}{2}}
$$

This rate is the same as the one for the critical NLS (Landman et al. [17], see also [22]). In Figure 6, we added as a dashed line the analytic blowup by the above formula, with $t_{*}$ at the value we found for our computation. It can be observed that the rate of blowup is recovered quite accurately.

Figure 7 shows contours of $|u|^{2}$ near the focus, and Figure 8 shows $|u|^{2}$ as surface plots at various times close to the blowup time. A distinct anisotropy can be observed.

Next we investigate the case $\chi=1$ (defocusing nonlinearity) and choose $\chi=1, \gamma=5$. In this case the second nonlinear term is causing the blowup. The corresponding $L^{\infty}$-norm of $u$ is shown in Figure 9 . In this case the 


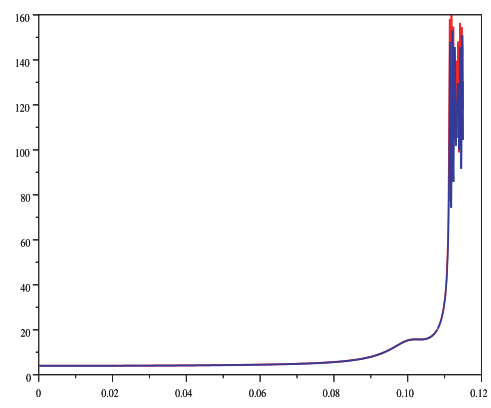

Figure 9. Blowup of defocusing ell.-ell. system $(\nu=1, \beta=5) . L^{\infty}$ norm of $|\Psi|$ for different time discretization steps. Blue: $\Delta t=5 \times 10^{-5}$, red: $\Delta t=2.5 \times 10^{-5}$.

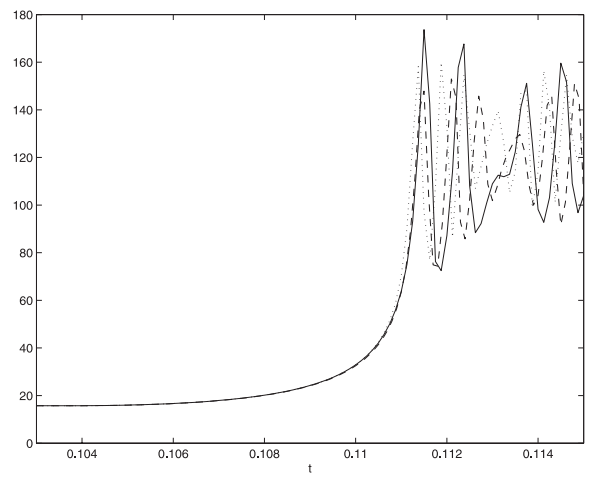

Figure 10. Blowup of defocusing ell.-ell. system. $L^{\infty}$ norm of $|\Psi|$ for various space and time discretization steps, zoom. dashed line: $\Delta x=0.0156, \Delta t=5 \times 10^{-5}$; dotted line: $\Delta x=0.0156, \Delta t=2.5 \times 10^{-5}$, solid: $\Delta x=0.00781, \Delta t=2.5 \times 10^{-5}$.

a)

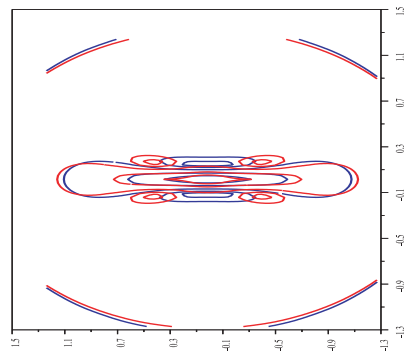

b)

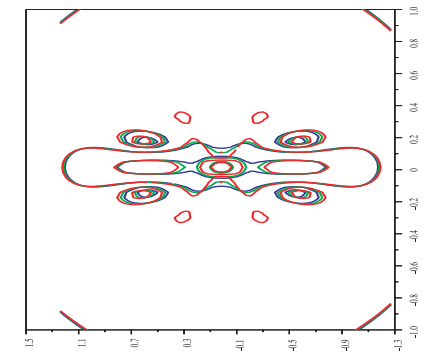

Figure 11. (a) Contour plot of position densities before blowup. $t=0.099, t=0.1045$.

(b) Contour plot of position densities close to blowup. $t=0.1091, t=0.1101, t=0.111$.

blowup is more complicated, there is a first concentration at $t=0.1$ before the blowup at $t=0.1115$. A much finer time and space resolution is needed to capture this blowup correctly. Figure 10 shows the maximum values of $|u|^{2}$ around the blowup time for several resolutions.

Figure 11 shows contours of $|u|^{2}$ close to the blowup, Figure 12 shows surface plots. We can see that there is more anisotropy in the results for this case than in the previous, NLS-dominated case. 
a)

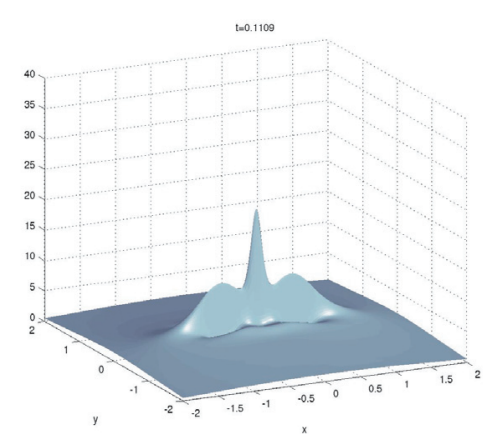

c)

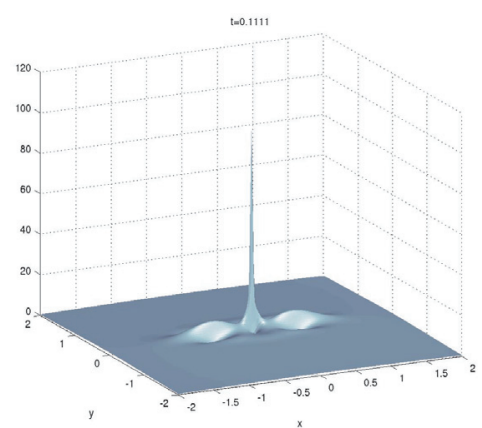

b)

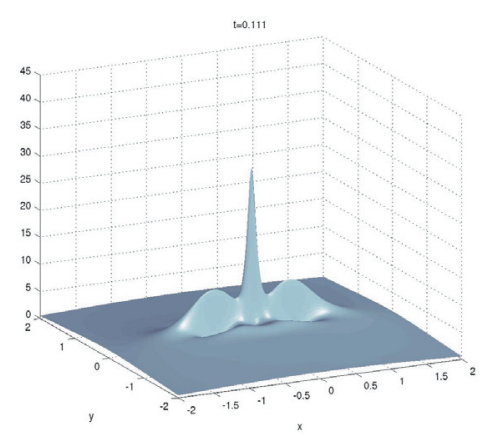

d)

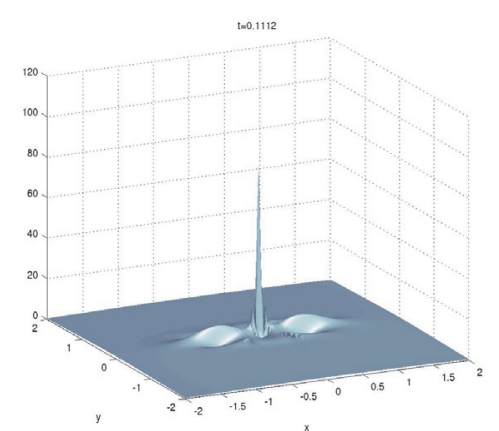

Figure 12. Position density at several times close to blowup. (a) $t=0.1109$, (b) $t=0.111$, (c) $t=0.1111,(\mathrm{~d}) t=0.1112$.

\subsubsection{Multi-Focusing}

For the focusing critical NLS, according to a result of Merle [18] it is possible to construct a solution which blows up exactly at a prescribed set of points. We state his result. Let, in $\mathbb{R}^{d}$ with a general dimension $d$, $R_{0}, R_{1}, \ldots$ be the infinite sequence of radial solutions to the equation

$$
\Delta R-R+R^{\frac{4}{d}+1}=0
$$

such that $R_{k}$ has exactly $k$ nodes as a function of $r$ and decreases exponentially at infinity.

Theorem 3.1 [18]. Let $\mathbf{x}_{1}, \ldots, \mathbf{x}_{k}$ be given in $\mathbb{R}^{d}$. There is a constant $\omega_{0}$ such that for any constants $\omega_{1}, \ldots, \omega_{k}$ all strictly larger than $\omega_{0}$, there exists a solution $\psi$ of the critical (focusing) NLS that blows up in a finite time $t_{*}$ such that:

(1) the set of blowup points in $L^{2+4 / d}$ and $H^{1}$ is $\left\{\mathbf{x}_{1}, \ldots, \mathbf{x}_{k}\right\}$;

(2) for $i=1, \ldots, k$ and all $A$ such that the balls $B_{i}=B\left(\mathbf{x}_{i}, A\right)$ are disjoint, $\lim _{t \rightarrow t_{*}}\|\psi(t)\|_{L^{2}\left(B_{i}\right)}=\left\|R_{i}\right\|_{L^{2}}$;

(3) $\lim _{t \rightarrow t_{*}}\|\psi(t)\|_{L^{2}(\bar{B})}=0$, where $\bar{B}=\mathbb{R}^{d} \backslash \cup_{i=1, \ldots, k} B_{i}$.

In addition, there is a constant $\gamma>0$ such that on $\left[0, t_{*}\right)$,

$$
\left\|\psi(t)-\sum_{i=1}^{k} \frac{1}{\left|\left(t_{*}-t\right) \omega_{i}\right|^{d / 2}} \mathrm{e}^{\frac{-i}{\left(t_{*}-t\right) \omega_{i}^{2}}+\frac{i|\mathbf{x}|^{2}}{4\left(t_{*}-t\right)}} R_{i}\left(\frac{\mathbf{x}-\mathbf{x}_{i}}{\omega_{i}\left(t_{*}-t\right)}\right)\right\|_{L^{2+4 / d}} \leq \mathrm{e}^{-\frac{\gamma}{t_{*}-t}}
$$


a)

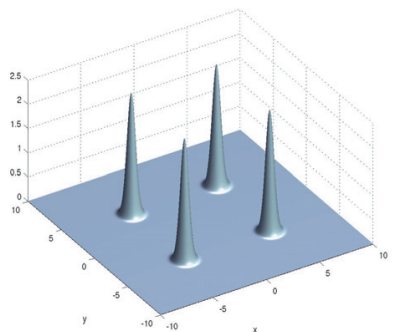

c)

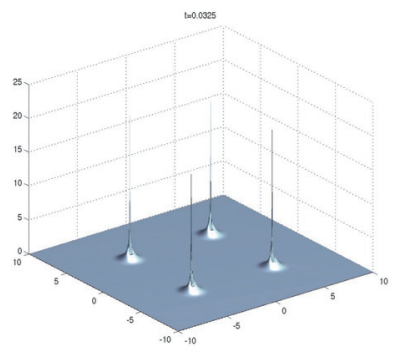

b)

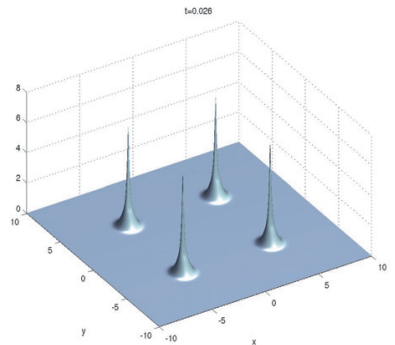

d)

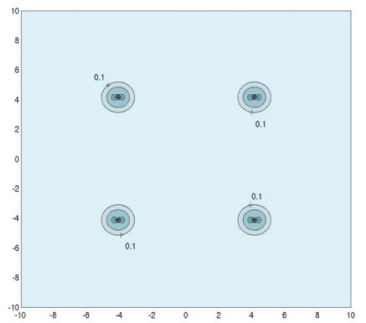

FIgURE 13. Multi-focusing solution. (a) Initial data, (b) result at intermediate time, (c) shortly after blowup time, (d) contour plot of result of (c), lowest contour level at 0.1.

Papanicolaou et al. [21] proved that there is a ground state solution to the focusing E-E DS system (3.1). Since there is only one ground state, we decide instead of calculating with the exact ground-state profile to choose a test profile $Q(\mathbf{x})=\exp \left(-|\mathbf{x}|^{2}\right)$, which is radial and decaying sufficiently fast. In order to realize the asymptotics described by the above result, we take the initial data as

$$
u_{I}=\sum_{j=1}^{k} \frac{1}{\tilde{t} \omega} \mathrm{e}^{-\frac{i}{\hat{t} \omega^{2}}+i \frac{|\mathbf{x}|^{2}}{4 t}} Q\left(\frac{\mathbf{x}-\mathbf{x}_{j}}{\tilde{t} \omega}\right)
$$

where $\tilde{t}$ is an estimate of the blowup time and $\omega$ a constant (and $\mathbf{x}=(x, y) \in \mathbb{R}^{2}$ ). We solve the equation on the domain $[-10,10]^{2}$, and choose the focus points $\mathbf{x}_{1}=(4,4), \mathbf{x}_{2}=(-4,4), \mathbf{x}_{3}=(-4,-4), \mathbf{x}_{4}=(4,-4)$ and the constants $\tilde{t}=0.03, \omega=13.3$. We find that blowup occurs at $t_{*}=0.032$. Figure 13 shows the position densities at initial time, at an intermediate time and at the blowup. We can see that the solution blows up exactly at the four chosen points.

\section{Conclusion}

Our numerical study of the Davey-Stewartson (DS) model is based on a "time-splitting spectral method", also called "Fourier split step method" by previous authors. Introduced by Tappert et al. in [13], this method has first been used for the hyperbolic-elliptic (H-E) DS equations by White, Weideman [23] ten years ago.

The new code we use has been developed independently of this previous work and takes advantage of today's computing power, in particular parallelization. 
The time-splitting spectral scheme has a number of advantages which make it suitable for Schrödinger type equations such as (cubic) NLS and DS. The basis of our code, developed by Bao and Jin, has already been successfully applied for "semiclassical" Nonlinear Schrödinger equations [4,5].

We not only study H-E DS systems, like White/Weidemann, but also E-E DS systems. Exact soliton type solutions of H-E DS can be recovered accurately, eliminating numerical dispersion effects which appear in earlier results of $[7,23]$. For the finite time blowup solution of H-E DS, the blowup rate can be recovered.

For the E-E DS systems, we study finite time blowup of the focusing and defocusing equations. In the focusing case the analytic blowup rate is recovered accurately and blowup profiles are presented, in full agreement to the results in [7]. The blowup mechanism in this case is very similar to the one of critical focusing NLS. In the defocusing case, however, a resolution fine enough to approximate the blowup is new. High numerical resolution in the results admits a detailed representation of blowup profiles in both cases.

For the first time, we investigate the phenomenon of simultaneous blowup at a predefined, exact number of points. This is known for focusing NLS; we find that focusing E-E DS can show the same behaviour.

Continuation of the solution after the blowup time is an interesting challenge for numerical simulations that seems to be in reach with our new code. Further work in this direction will be performed.

Acknowledgements. Financial support by the Austrian START award project (FWF, contract No. Y-137-TEC), by the Wissenschaftskolleg (doctoral school) "Differential Equations" (FWF, contract No. W8) as well as the European network HYKE, funded by the EC as contract HPRN-CT-2002-00282, is acknowledged.

\section{REFERENCES}

[1] M.J. Ablowitz and P.A. Clarkson, Solitons, nonlinear evolution equations and inverse scattering. Cambridge University Press, London Math. Soc. Lect. Note Series 149 (1991).

[2] M.J. Ablowitz and H. Segur, Solitons and the inverse scattering transform. SIAM Stud. Appl. Math., SIAM, Philadelphia 4 (1981).

[3] V.A. Arkadiev, A.K. Pogrebkov and M.C. Polivanov, Inverse scattering transform method and soliton solutions for the Davey-Stewartson II equation. Physica D 36 (1989) 189-196.

[4] W. Bao, S. Jin and P.A. Markowich, Time-splitting spectral approximations for the Schrödinger equation in the semiclassical regime. J. Comp. Phys. 175 (2002) 487-524.

[5] W. Bao, N.J. Mauser and H.P. Stimming, Effective one particle quantum dynamics of electrons: a numerical study of the Schrödinger-Poisson-X $\alpha$ model. CMS 1 (2003) 809-831.

[6] C. Besse, Schéma de relaxation pour l'équation de Schrödinger non linéaire et les systèmes de Davey et Stewartson. $C$. $R$. Acad. Sci. Paris I 326 (1998) 1427-1432.

[7] C. Besse and C.H. Bruneau, Numerical study of elliptic-hyperbolic Davey-Stewartson system: dromions simulation and blow-up. Math. Mod. Meth. Appl. Sci. 8 (1998) 1363-1386.

[8] C. Besse, B. Bidégaray and S. Descombes, Order estimates in time of the splitting methods for the nonlinear Schrödinger equation. SIAM J. Numer. Anal. 40 (2002) 26-40.

[9] S. Descombes, Convergence of a splitting method of high order for reaction-diffusion systems. Math. Comp. 70 (2001) $1481-1501$.

[10] V.D. Djordjević and L.G. Redekopp, On two-dimensional packets of capillary-gravity waves. J. Fluid Mech. 79 (1977) 703-714.

[11] J.M. Ghidaglia and J.C. Saut, On the initial value problem for the Davey-Stewartson systems. Nonlinearity 3 (1990) 475-506.

[12] M. Guzmán-Gomez, Asymptotic behaviour of the Davey-Stewartson system. C. R. Math. Rep. Acad. Sci. Canada 16 (1994) 91-96.

[13] R.H. Hardin and F.D. Tappert, Applications of the split-step Fourier method to the numerical solution of nonlinear and variable coefficient wave equations. SIAM Rev. Chronicle 15 (1973) 423.

[14] N. Hayashi, Local existence in time solutions to the elliptic-hyperbolic Davey-Stewartson system without smallness condition on the data. J. Anal. Math. LXXIII (1997) 133-164.

[15] N. Hayashi and H. Hirata, Global existence and asymptotic behaviour of small solutions to the elliptic-hyperbolic DaveyStewartson system. Nonlinearity 9 (1996) 1387-1409.

[16] N. Hayashi and J.C. Saut, Global existence of small solutions to the Davey-Stewartson and Ishimori systems. Diff. Int. Eq. 8 (1995) $1657-1675$. 
[17] M.J. Landman, G.C. Papanicolaou, C. Sulem and P.-L. Sulem, Rate of blowup for solutions of the Nonlinear Schrödinger equation at critical dimension. Phys. Rev. A 38 (1988) 3837-3843.

[18] F. Merle, Construction of solutions with exactly k blowup points for the Schrödinger equation with critical nonlinearity. Comm. Math. Phys. 129 (1990) 223-240.

[19] K. Nishinari, K. Abe and J. Satsuma, Multidimensional behaviour of an electrostatic ion wave in a magnetized plasma. Phys. Plasmas 1 (1994) 2559-2565.

[20] T. Ozawa, Exact blow-up solutions to the Cauchy problem for the Davey-Stewartson systems. Proc. R. Soc. A 436 (1992) $345-349$.

[21] G.C. Papanicolaou, C. Sulem, P.-L. Sulem, X.P. Wang, The focusing singularity of the Davey-Stewartson equations for gravity-capillary surface waves. Physica D 72 (1994) 61-86.

[22] C. Sulem and P.-L. Sulem, The Nonlinear Schrödinger Equation: Self-Focusing and Wave Collapse. Springer, New York (1999)

[23] P.W. White and J.A.C. Weideman, Numerical simulation of solitons and dromions in the Davey-Stewartson system. Math. Comput. Simul. 37 (1994) 469-479.

To access this journal online: www.edpsciences.org 